\title{
The rise of barefoot running
}

K ate Kift took up running two years ago to improve her work out that way at first. Within a few months, she developed stress fractures in her heels. Her doctor recommended that she stop running. But Kift, who is 37 years of age, didn't stop. Instead, she ditched her running shoes, joining the growing community of people who believe running barefoot is better for the body than running in supportive footwear.

"It became quite evident that, when I was in shoes, I was striking severely on my heels," says Kift.

People who run barefoot tend to have shorter strides and land on the front or middle of their feet instead of their heels. Advocates of barefoot running say this style of running decreases the severity of impact with the ground and reduces injuries. But not everyone agrees.

Critics of barefoot running, including many podiatrists, note that there is no scientific evidence that indicates running barefoot is better than running in shoes, and say that even if running barefoot reduces some types of injuries it may cause other types of harm, such as puncture wounds on the soles and stress fractures in the metatarsals.

Dr. Michael Nirenberg, a podiatrist who practises in Crown Point, Indiana, is not among those critics. Nirenberg has been a runner off and on throughout his life, and has suffered from plantar fasciitis, a painful inflammation of the main ligament in the foot's arch. This led him to conduct research on ways to strengthen the muscles in feet, which in turn led him to become a fan of going barefoot.

"I started reading about our feet and shoes and supportive shoes. I became intrigued with the idea that once you support the arch of the foot, you don't use your foot muscles as much," says Nirenberg, who writes about barefoot running and other topics on his blog (www.americaspodiatrist.com). "If you

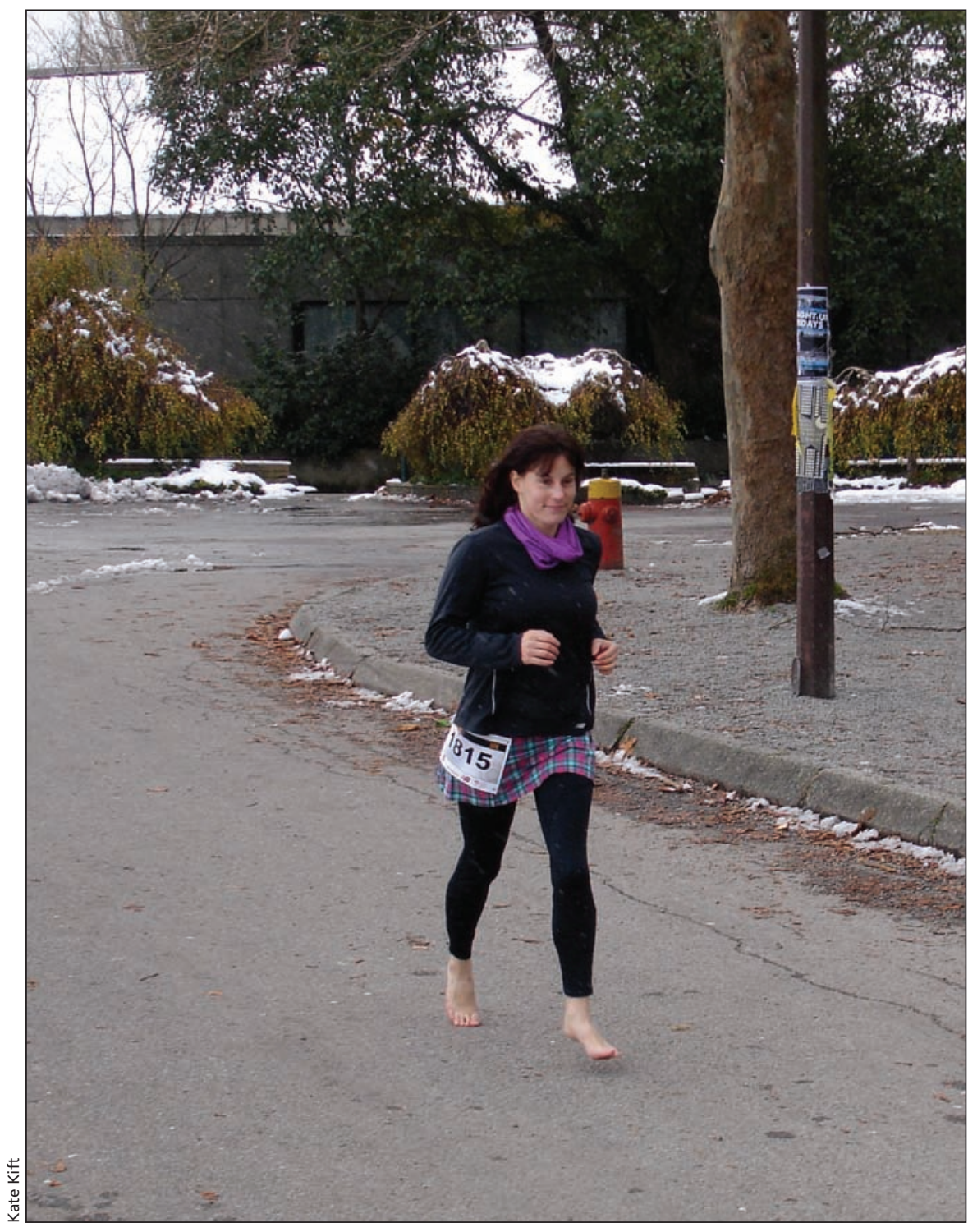

Kate Kift of British Columbia shed her sneakers and took up barefoot running after developing stress fractures in her heels and is now injury-free.

start doing barefoot activity, be it running or walking, you start to build up the muscles in your feet."

His views aren't common in his profession, which tends to focus on using orthotics to correct foot problems. Orthotics do relieve pain quicker, Nirenberg acknowledges, but he recommends that people with foot problems transition over time to lesssupportive footwear, and eventually incorporate some barefoot activity into their lives. As for barefoot running, Nirenberg agrees with the critics that there is no proof of its benefits at least, not yet.

"Right now, there is no proof that running barefoot is better for you, but there is a lot of research that is leaning in that direction," he says.

Still, despite the paucity of research in this area, the barefoot running trend is growing. The Barefoot Runners Society, founded in the United States 
just over a year ago, has 1500 members (www.barefootrunners.org). And more people are becoming interested in running sans shoes everyday, says Tamara Gerken, the society's president and cofounder. Gerken threw away her running shoes after developing a painful condition called Morton's neuroma.

"Like many people suffering with running shoe-related injuries or conditions, I came to a realization that I wasn't the one who was defective, it was the shoes," Gerken writes in an email. "Running should be carefree and natural, not bound and controlled."

Kift, who lives in British Columbia and is president of the newly formed Canadian chapter of the Barefoot Runners Society, is now injury free. Six months after being diagnosed with heel fractures, she ran a half-marathon. She plans on running many more races next year, including a 50-kilometre trail race. Kift writes about barefoot running on her blog (barefootkatiek.blogspot.com), and says that making the switch from the traditional running style may cause some initial discomfort, but it eventually makes people into more careful runners.

"There is always transitional pain during the period of transition when your body is running in a completely different way," says Kift. "But when you run in shoes, you zone out a little bit. When running barefoot, you have to be very aware of what's in front of you, very aware of the terrain and very aware of the environment."

The positive reports of the health benefits of running with naked feet, however, are all anecdotal. Though interest in research into barefoot running is growing, much like the trend itself, there is little in the way of hard evidence to back up the enthusiastic stories of barefoot runners.

"There is not a shred of research that indicates running barefoot or running in minimalist shoes reduces injuries," says Dr. Kevin Kirby, an adjunct associate professor at the California School of Podiatric Medicine in Oakland. "There is also no research that indicates running shoes reduce injuries. It's a wash as far as research is concerned."

Kirby, who has been a sports podiatrist for 25 years, is himself an accomplished runner, having completed more than a dozen marathons, with a morethan-respectable personal best of two hours and 28 minutes. He even has experience in barefoot running. In university, where he ran competitively, he sometimes trained in bare feet on the grass of a baseball field. For advanced runners, says Kirby, incorporating barefoot running into their training can be useful.

As for beginners shedding their shoes because of running-induced injuries, Kirby is skeptical. Often, a person picks up running after a few years of sedentary existence and trains too hard, pounding the pavement with reckless zeal while carrying 10 or more kilograms of excess body weight. Injuries often ensue, and running shoes make a convenient scapegoat.

Running barefoot on extremely hot pavement or in extremely cold weather might also damage the soles of your feet, Kirby warns. Then there are nails and glass and pebbles and other objects that can puncture the soles of feet or lead to stubbed toes. And even if heel strikes are eliminated by running barefoot, shorter strides means the feet hit the ground more often, so what's gained by reducing the force of impact might be offset by the increase in frequency of impact. The benefits of barefoot running are being oversold, says Kirby, and it is novelty that's driving the trend.

"It's another fad, something that drives a new cycle," says Kirby. "It's interesting and it's something to talk about. In running, there's not much you can do differently, so this generates discussion."

That discussion can sometime get heated. Runners often trade training tips and seek advice on Internet forums, and barefoot advocates are no exception. If anything, barefoot converts are so enthusiastic that they twist the findings of research papers to support their claims, says Craig Payne, a senior lecturer in the department of podiatry at La Trobe University in Melbourne, Australia.

"The barefoot running community have an appalling track record at how they misinterpret, misuse and misquote research," Payne writes in an email. "The simple facts are that not one risk factor study on running injuries has linked high impacts to running injuries, yet the barefoot running community claim that the evidence shows this and consider high impacts as the cause of all injuries."

A recent paper frequently cited by barefoot runners states that "fore-footand mid-foot-strike gaits were probably more common when humans ran barefoot or in minimal shoes, and may protect the feet and lower limbs from some of the impact-related injuries now experienced by a high percentage of runners" (Nature 2010;463:531-5).

On their website, however, the authors of the paper make it clear that they are not advocating for barefoot running, stating that they have no data on how people should run and calling for controlled studies on this topic (www .barefootrunning.fas.harvard.edu).

"People are jumping to conclusions on both sides of the debate, but we must rely on evidence," says Dan Lieberman, one of the paper's authors and a professor of human evolutionary biology at Harvard University in Cambridge, Massachusetts. "The media is distorting the science or just leaving it out. The key thing is not being barefoot, but using a barefoot style, and not colliding into the ground with your heels." - Roger Collier, CMAJ

CMAJ 2011.DOI:10.1503/cmaj.109-3745 